\title{
Utility of small biopsies for diagnosis of lung nodules: doing more with less
}

\author{
Sanjay Mukhopadhyay \\ Department of Pathology, State University of New York Upstate Medical University, Syracuse, NY, USA
}

\begin{abstract}
Small biopsies obtained by core needle or bronchoscopy are commonly used for diagnosis of lung nodules. This review provides guidance in two key areas of interpretation of small lung biopsies. The first part answers common questions regarding the immunohistochemical subclassification of non-small cell lung carcinomas that cannot be classified by standard criteria on hematoxylin-eosin-stained sections of small lung biopsies. The second part discusses common benign entities that can be diagnosed in core needle biopsies of lung nodules, such as granulomatous inflammation, parenchymal scars and organizing pneumonia. The approach to cases in which malignant cells are absent and features of a specific benign diagnosis are lacking is also addressed. Modern Pathology (2012) 25, S43-S57; doi:10.1038/modpathol.2011.153
\end{abstract}

Keywords: core needle biopsy; immunohistochemistry; lung cancer classification; napsin A; non-small cell lung carcinoma; transbronchial biopsy; TTF-1

Histological examination is the gold standard for definitive diagnosis of lung nodules (masses). Although resection of lung nodules is ideal for diagnosis, small biopsies-including core needle, transbronchial and endobronchial biopsies-are often used to obtain a tissue diagnosis without subjecting patients to the risks associated with a surgical procedure. For pathologists, the use of small biopsies raises several issues related to diagnostic limitations imposed by partial sampling, the role of immunohistochemical markers in diagnosing malignant tumors and the approach to cases that show non-specific findings. The first part of this review deals with malignant diagnoses and focuses on an area that has recently emerged as an important task for pathologists who interpret small lung biopsies, ie, subclassification of non-small cell lung carcinomas using immunohistochemical markers. The second part of the article reviews specific benign diagnoses that can be made in core needle biopsies of lung nodules. The review is structured in a 'frequently asked questions' format.

Correspondence: Dr S Mukhopadhyay, MD, Department of Pathology, State University of New York Upstate Medical University, 750 E. Adams Street, Syracuse, NY 13210, USA.

E-mail: mukhopas@upstate.edu

Received 3 July 2011; accepted 6 September 2011

\section{A generic diagnosis of 'non-small cell lung carcinoma' was sufficient in the past. Why do we now need to subclassify non-small cell lung carcinomas?}

In the past, there was no clinical demand for subclassifying non-small cell lung carcinomas because subclassification had no impact on therapy. Patients with advanced-stage non-small cell lung carcinoma who received chemotherapy were typically treated with a 'platinum doublet,' such as cisplatin plus gemcitabine irrespective of histological subtype. At present, in contrast, subclassification of non-small cell lung carcinoma has significant treatment implications, especially for advancedstage tumors for which chemotherapy is being considered. Consequently, pathologists may be asked to determine whether a given non-small cell lung carcinoma is an adenocarcinoma or squamous cell carcinoma. The demand for subtyping is driven by oncologists, who now have several new targeted therapies in their armamentarium the efficacy (and in one case, safety) of which varies by histological subtype. In particular, the folate inhibitor pemetrexed is efficacious in the treatment of adenocarcinomas and poorly differentiated non-small cell carcinomas, but not squamous cell carcinomas. ${ }^{1}$ The epidermal growth factor receptor (EGFR) inhibitors erlotinib and gefitinib are of greatest benefit in EGFR mutation-positive tumors, which are predominantly adenocarcinomas. ${ }^{2}$ The vascular 
endothelial growth factor inhibitor bevacizumab has been associated with an increased incidence of lifethreatening hemoptysis in patients with squamous cell carcinomas but not adenocarcinomas. ${ }^{3}$ Finally, crizotinib, an inhibitor of anaplastic lymphoma kinase (ALK), is efficacious in tumors with EML4ALK fusions, which occur predominantly in adenocarcinomas. ${ }^{4}$ In each of these examples, histological subtyping of non-small cell lung carcinoma impacts selection or exclusion of specific targeted agents.

\section{Is immunohistochemistry necessary for subtyping non-small cell lung carcinomas?}

The answer to this question depends on differentiation of the tumor and the type of specimen in which it is diagnosed. At present, morphological assessment on hematoxylin-eosin (H\&E)-stained sections remains the gold standard for subclassifying nonsmall cell lung carcinomas in lung resections. Subclassification in small biopsies is more problematic as such specimens do not sample the entire tumor. Even in small biopsies, however, there is universal agreement that most non-small cell lung carcinomas can be classified on H\&E-stained slides alone without using any immunohistochemical stains. In particular, tumors that form glands or contain mucin can be diagnosed as adenocarcinoma, whereas those that produce keratin or contain intercellular bridges are squamous cell carcinomas. Examples of non-small cell lung carcinomas that can be subclassified without immunohistochemistry are shown in Figure 1. Immunohistochemical stains are required only for those tumors that are "poorly differentiated', ie, those in which standard H\&Ebased morphological criteria used for subclassifying non-small cell lung carcinomas cannot be identified (Figure 2). Stated another way, immunohistochemical stains are not required for subclassifying well-differentiated or moderately differentiated non-small cell lung carcinomas. The precise cutoff at which a tumor should be considered unclassifiable on H\&E-stained slides is difficult to define, and in practice, there is considerable variation in the point at which individual pathologists feel comfortable that they have identified features convincing enough to render a diagnosis based on H\&E-stained slides alone. Consequently, some variability in the threshold for the use of immunohistochemistry is inevitable. Nevertheless, tumors that are readily classifiable on H\&E-stained slides should not be stained with immunohistochemical markers. Instead, biopsied tissue in such cases should be conserved in the event that the tumor proves to be
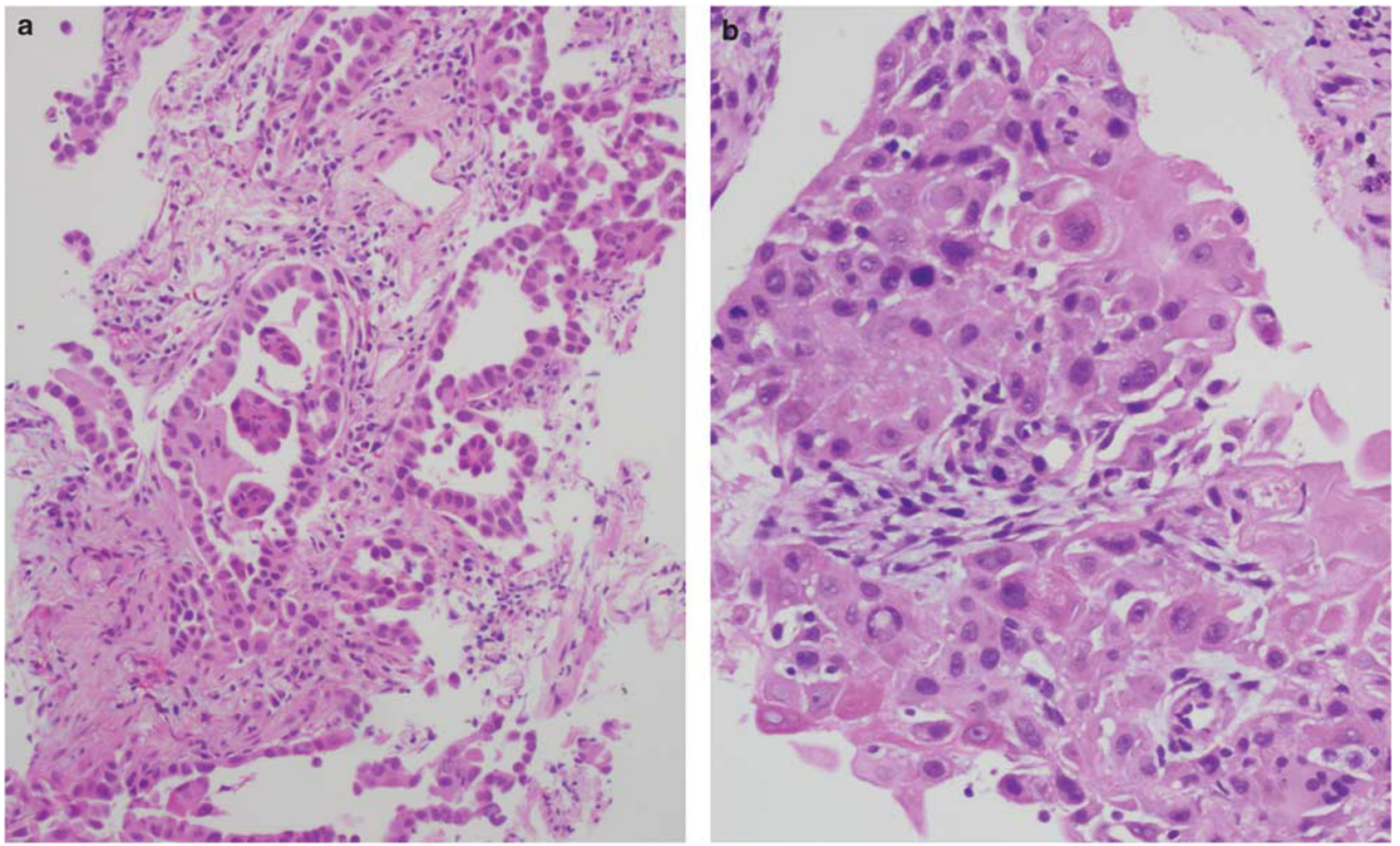

Figure 1 Examples of non-small cell lung carcinomas that do not require immunohistochemical stains for diagnosis, even in a small biopsy. (a) Well-differentiated adenocarcinoma. Glands are easily identifiable. (b) Moderately differentiated squamous cell carcinoma. Intercellular bridges are present and there is a hint of keratinization. 


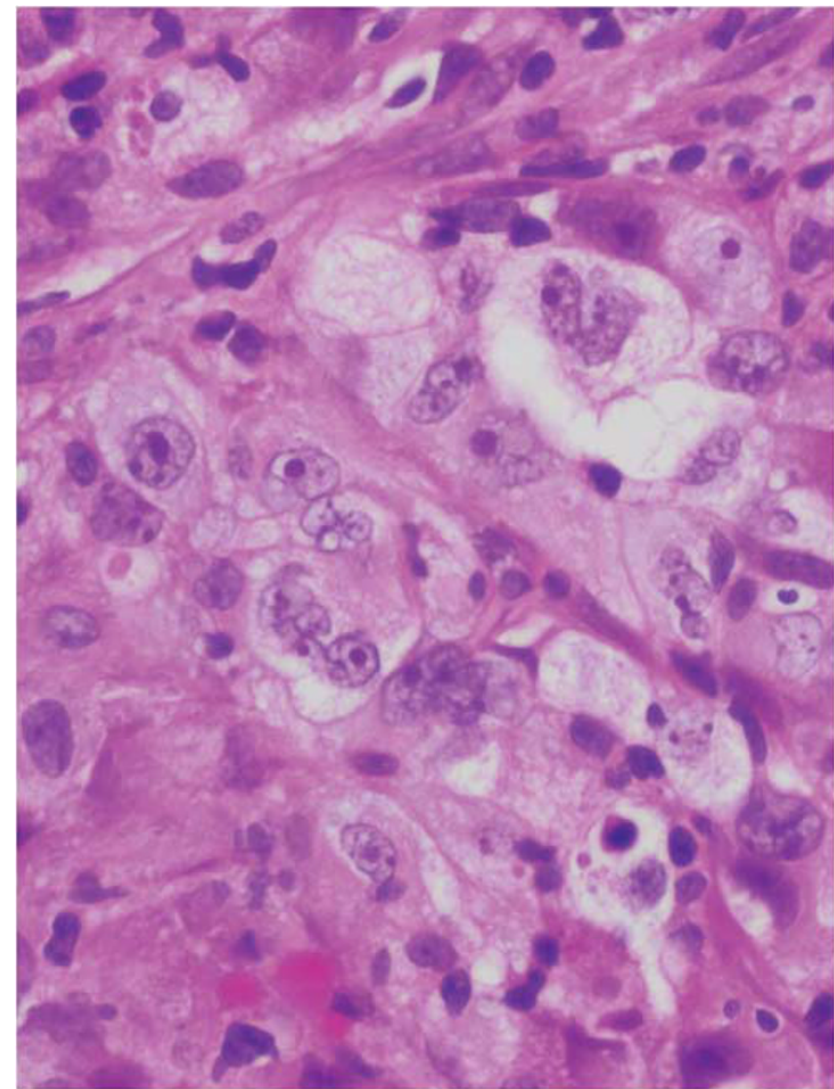

Figure 2 Example of a poorly differentiated non-small cell lung carcinoma that requires immunohistochemical stains for subclassification in a small biopsy. There is no evidence of gland formation, mucin production, intercellular bridges or keratinization.

unresectable and tissue is required for molecular testing (EGFR or EML4-ALK) in the future.

\section{Why is immunohistochemistry being used for diagnosis if H\&E morphology is the gold standard?}

Some non-small cell lung carcinomas are too poorly differentiated to be accurately subclassified by standard H\&E criteria, especially in small biopsies. Data from several previous immunohistochemical studies seemed to indicate that immunohistochemistry, although promising, lacked the specificity required for accurate subclassification. However, recent immunohistochemical studies that have focused on poorly differentiated non-small cell carcinomas in small biopsies or cytology specimens with resected tumors as the gold standard have shown that some markers, especially when used in combination, are indeed useful and accurate in subclassifying poorly differentiated non-small cell lung carcinomas. ${ }^{5-8}$ As subclassification using these markers shows excellent correlation with the gold standard H\&E-based diagnosis on resected tumors, immunohistochemistry has become accepted as a reliable technique for subclassification when diagnostic features on H\&E morphology are absent in a small biopsy. To summarize the current state of non-small cell lung carcinoma subclassification, H\&E-based criteria remain the gold standard for subclassifying resected tumors. H\&E is also sufficient for subclassifying tumors in small biopsies that show glands, mucin, intercellular bridges or keratinization. However, for non-small cell lung carcinomas in small biopsies in which these features cannot be identified, immunohistochemistry is the sole basis for subclassification.

\section{Which immunohistochemical markers are useful in the subclassification of non-small cell lung carcinomas and which are too non-specific to be of utility?}

The most useful immunohistochemical markers for subclassifying poorly differentiated non-small cell lung carcinomas in small biopsies are TTF-1, napsin A, p63 and CK5 $/ 6 .{ }^{5}$ These markers are fairly sensitive and specific individually, but the advantage of a panel composed of all four is that it provides back-up markers that compensate for the shortcomings of each individual marker. Napsin A, eg, is useful in cases in which TTF-1 is focal, weak or difficult to interpret for technical reasons. Furthermore, although napsin A is slightly less sensitive for poorly differentiated adenocarcinomas than TTF-1, it is valuable in occasional adenocarcinomas that are napsin A-positive but TTF-1-negative. ${ }^{8-10}$ Finally, napsin A stains adenocarcinomas but not small cell carcinomas, ${ }^{9}$ a fact that can be helpful when the differential diagnosis of a TTF-1positive poorly differentiated carcinoma includes small cell carcinoma. Similarly, although p63 is more sensitive for squamous cell carcinomas than CK5/6, the latter is useful when p63 staining is focal, weak or equivocal. ${ }^{7}$ In samples for which cellularity is very low, it has been suggested that a more limited panel composed of TTF-1 and p63 can be used in most cases to conserve the tissue in the event that it is required for molecular studies. ${ }^{7}$ Similar concerns have been cited by a consensus panel in recommending that the panel be limited to TTF-1 and p63, with or without a mucin stain. ${ }^{11}$ To date, however, there is no evidence that using one or two additional sections (ie, a 4-marker panel vs a 3- or a 2-marker panel) for immunohistochemistry diminishes the yield of subsequent molecular studies. Indeed, one recent study using transbronchial needle aspirates for non-small cell lung carcinoma subclassification showed that DNA sufficient for EGFR mutation testing could be extracted from all cases in which a 4-marker panel had been used. ${ }^{11}$

Other markers used in the past, such as CK7 for adenocarcinoma and $34 \beta \mathrm{E} 12$ for squamous cell carcinoma, are highly sensitive but too non-specific 
to be useful. CK7 is positive in virtually all poorly differentiated adenocarcinomas but also stains $60 \%$ of poorly differentiated squamous cell carcinomas (Figure 3). ${ }^{5}$ Similarly, 34 $\beta$ E12 stains not only $100 \%$ of poorly differentiated squamous cell carcinomas but also $60 \%$ of poorly differentiated adenocarcinomas. ${ }^{5,12}$ Therefore, positivity for either CK7 or $34 \beta$ E12 is of no utility for non-small cell lung
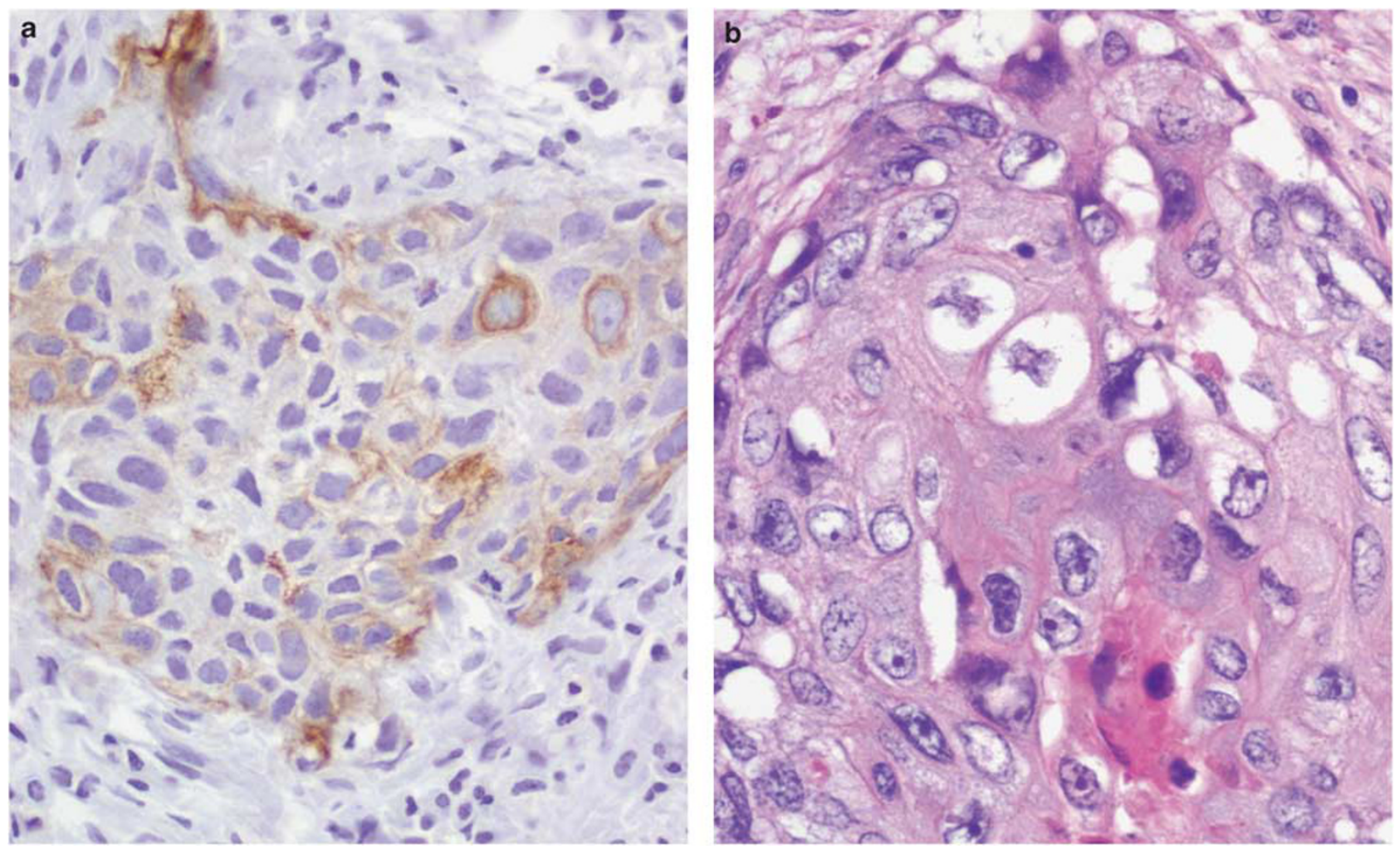

Figure 3 CK7 staining in poorly differentiated squamous cell carcinoma. (a) Small biopsy showing a poorly differentiated non-small cell carcinoma. The tumor is positive for CK7. (b) High magnification of the resected tumor shows intercellular bridges, confirming a diagnosis of squamous cell carcinoma. This case illustrates why CK7 is not a useful marker for subclassifying poorly differentiated nonsmall cell lung carcinomas.

Table 1 Algorithm for subclassification of poorly differentiated non-small cell lung carcinomas using immunohistochemical markers in small lung biopsies ${ }^{\mathrm{a}}$

\section{Adenocarcinoma markers} (TTF-1 and napsin A)
Squamous cell carcinoma markers (p63 and CK5/6)

TTF-1 or napsin A or both positive TTF-1 or napsin A or both positive Both negative

Both negative

Both negative

All four markers negative

TTF-1 and napsin A positive in one population of cells, p63 and CK5/6 positive in a separate population of cells
Both negative

p63 positive, CK5/6 negative

Both positive

p63 diffusely positive, CK5/6 negative

p63 focally positive, CK5/6 negative
Diagnosis on small biopsy

Poorly differentiated adenocarcinoma

Poorly differentiated adenocarcinoma

Poorly differentiated squamous cell carcinoma

Poorly differentiated squamous cell carcinoma

Poorly differentiated non-small cell carcinoma ${ }^{b}$

Poorly differentiated non-small cell carcinoma ${ }^{\mathrm{b}}$

Poorly differentiated adenosquamous carcinoma

\footnotetext{
${ }^{\mathrm{a}}$ These are modified from the study by Mukhopadhyay and Katzenstein. ${ }^{5}$

${ }^{\mathrm{b}}$ Alternative term: non-small cell lung carcinoma, not otherwise specified (NSCLC, NOS); review H\&E morphology, consider diagnoses other than carcinoma.
}

\footnotetext{
Figure 4 Immunohistochemical staining in poorly differentiated adenocarcinoma. (a) Small biopsy specimen containing a poorly differentiated non-small cell carcinoma that cannot be subclassified on H\&E-stained slides. Immunohistochemical staining of the biopsy shows that the tumor is positive for TTF-1 (b) and napsin A (c), but negative for p63 (d) and CK5/6 (e), diagnostic of poorly differentiated adenocarcinoma. The corresponding resection (f) shows H\&E features that confirm the immunohistochemical biopsy diagnosis. It must be noted that the poorly differentiated component corresponding to the biopsy (left) blends into a better differentiated component with glands (right) that allow its recognition as an adenocarcinoma.
} 


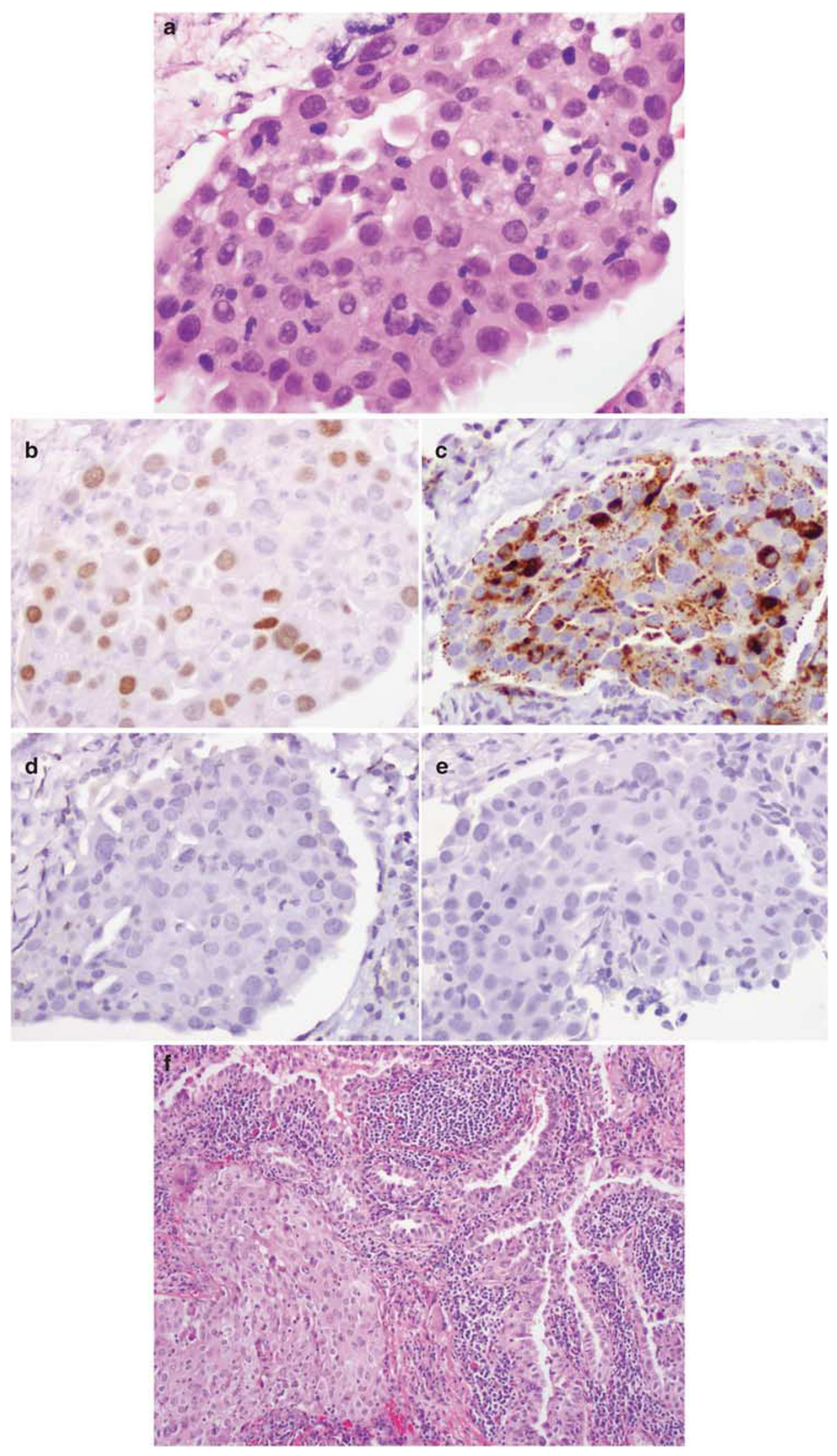



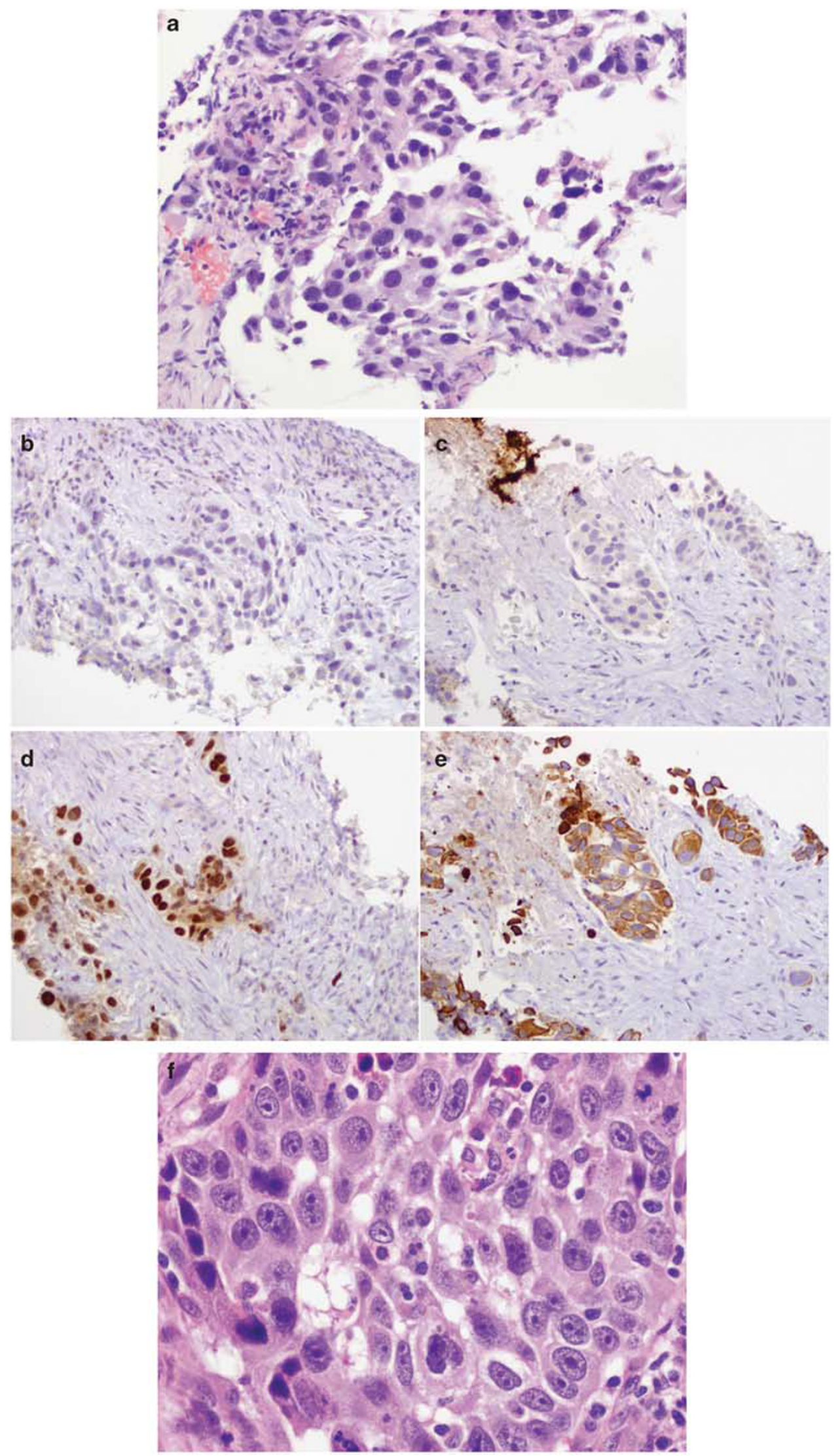
carcinoma subclassification. It is important to emphasize that although CK7 is unhelpful in subclassification of poorly differentiated non-small cell lung carcinomas, this does not diminish its utility in other settings. For example, if a tumor in a small lung biopsy is suspected to be metastatic based on morphology, clinical history or radiological findings, CK7 can be a valuable marker as part of a panel to determine a primary site.

Mucin stains such as mucicarmine or Alcian blueperiodic acid Schiff have been used in some studies as markers of adenocarcinoma. ${ }^{6,12}$ However, we do not use mucin stains in our panel as significant amounts of mucin are usually well visualized on H\&E-stained sections. Furthermore, mucin stains lack sensitivity for poorly differentiated adenocarcinomas $(23 \%)^{6}$ when compared with TTF-1 or napsin A (80 and 58\%, respectively). ${ }^{5}$

\section{How are various combinations of immuno- histochemical staining interpreted for subclassification of poorly differentiated non-small cell lung carcinomas?}

Our algorithm for subclassifying poorly differentiated non-small cell lung carcinomas using a panel of TTF-1, napsin A, p63 and CK5/6 is shown in Table 1. More than three-fourths of poorly differentiated non-small cell lung carcinomas can be accurately subclassified by immunohistochemistry using this approach. ${ }^{5}$ In most cases, interpretation of the panel is fairly straightforward. Either both glandular markers are positive and both squamous markers are negative, allowing a diagnosis of poorly differentiated adenocarcinoma (Figure 4), or both glandular markers are negative and both squamous markers are positive, diagnostic of poorly differentiated squamous cell carcinoma (Figure 5). Difficulties arise in cases that deviate from these classic staining patterns. Examples include tumors with positivity for TTF-1 and for p63, positivity for only one glandular marker (TTF-1 or napsin A) or positivity for p63 alone. Interpretation of cases, in which TTF-1 and p63 are both positive in the same tumor, is discussed in the next section. Tumors positive for one glandular marker only (either TTF-1 or napsin A) should be diagnosed as poorly differentiated adenocarcinoma. Interpretation of isolated p63 positivity depends on the proportion of tumor cells stained. As occasional poorly differentiated squamous cell carcinomas are p63 positive (diffuse) but CK5/6 negative, diffuse p63 positivity is diagnostic of poorly differentiated squamous cell carcinoma. However, isolated focal p63 positivity is non-specific, and such tumors should be considered unclassifiable. Other staining combinations (such as a positive CK5/6 with negative TTF-1, napsin A and p63) do occur occasionally but are uncommon, and there is insufficient evidence to subclassify them on small biopsies using immunohistochemical markers.

One pitfall in the use of immunohistochemistry is the presence of entrapped lung epithelium within tumor tissue. As both TTF-1 and napsin A stain alveolar epithelium, entrapped lung parenchyma in squamous cell carcinomas can be misinterpreted as TTF-1 or napsin A positivity in the tumor (Figure 6). Knowledge of this possibility, and careful attention to the size of the nuclei of entrapped cells will usually prevent this error. The phenomenon of entrapped lung epithelium probably accounts for at least some reports of TTF-1-positive

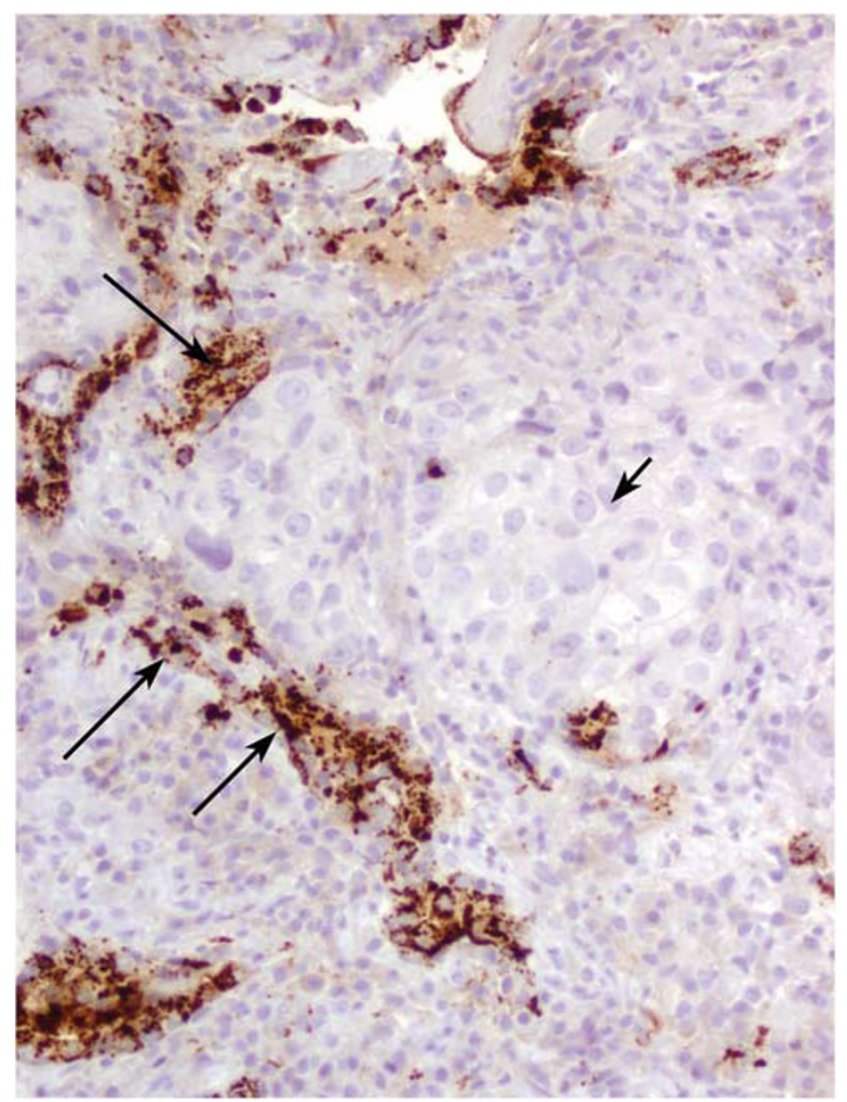

Figure 6 Entrapped lung epithelium: a potential pitfall. This squamous cell carcinoma (short arrow) contains entrapped lung epithelium that is positive for napsin A (long arrows). This finding should not be misinterpreted as napsin A positivity in a squamous cell carcinoma. Similar considerations apply to TTF-1 staining in the entrapped lung epithelium.

Figure 5 Small biopsy diagnosis of poorly differentiated squamous cell carcinoma by immunohistochemistry. (a) This small lung biopsy contains a non-small cell carcinoma that is not further classifiable on H\&E as it lacks glands, mucin, intercellular bridges and keratin. On the small biopsy, the neoplastic cells are negative for TTF-1 (b) and napsin A (c), but positive for p63 (d) and CK5/6 (e). The resected tumor (f) is recognizable as a squamous cell carcinoma by the presence of intercellular bridges. 

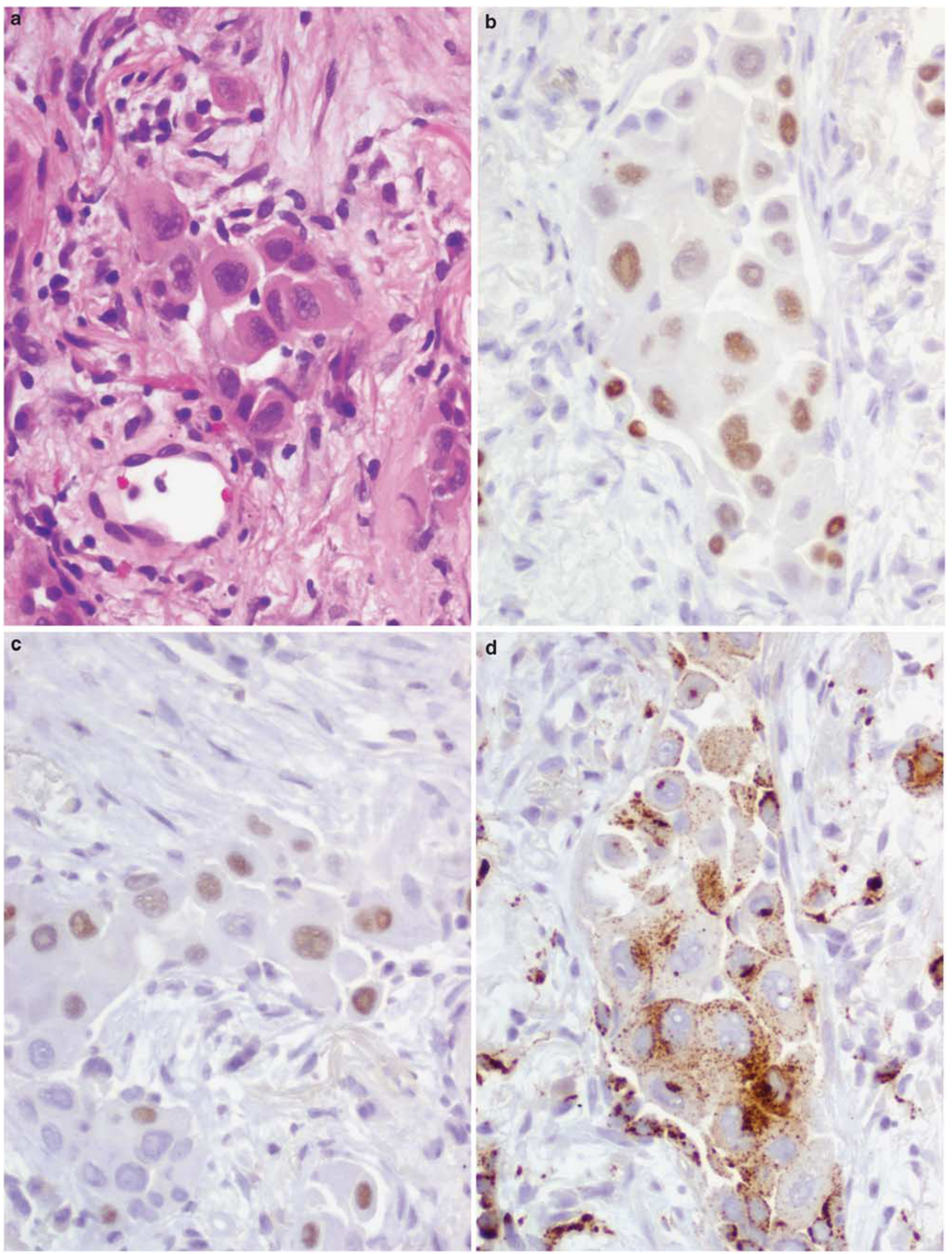

Figure 7 p63 positivity in poorly differentiated adenocarcinoma. (a) Poorly differentiated non-small cell lung carcinoma in a small biopsy. The tumor cells are positive for TTF-1 (b) and p63 (c), a combination that indicates a poorly differentiated adenocarcinoma. Napsin A (d) is also positive, helping to confirm the diagnosis. 


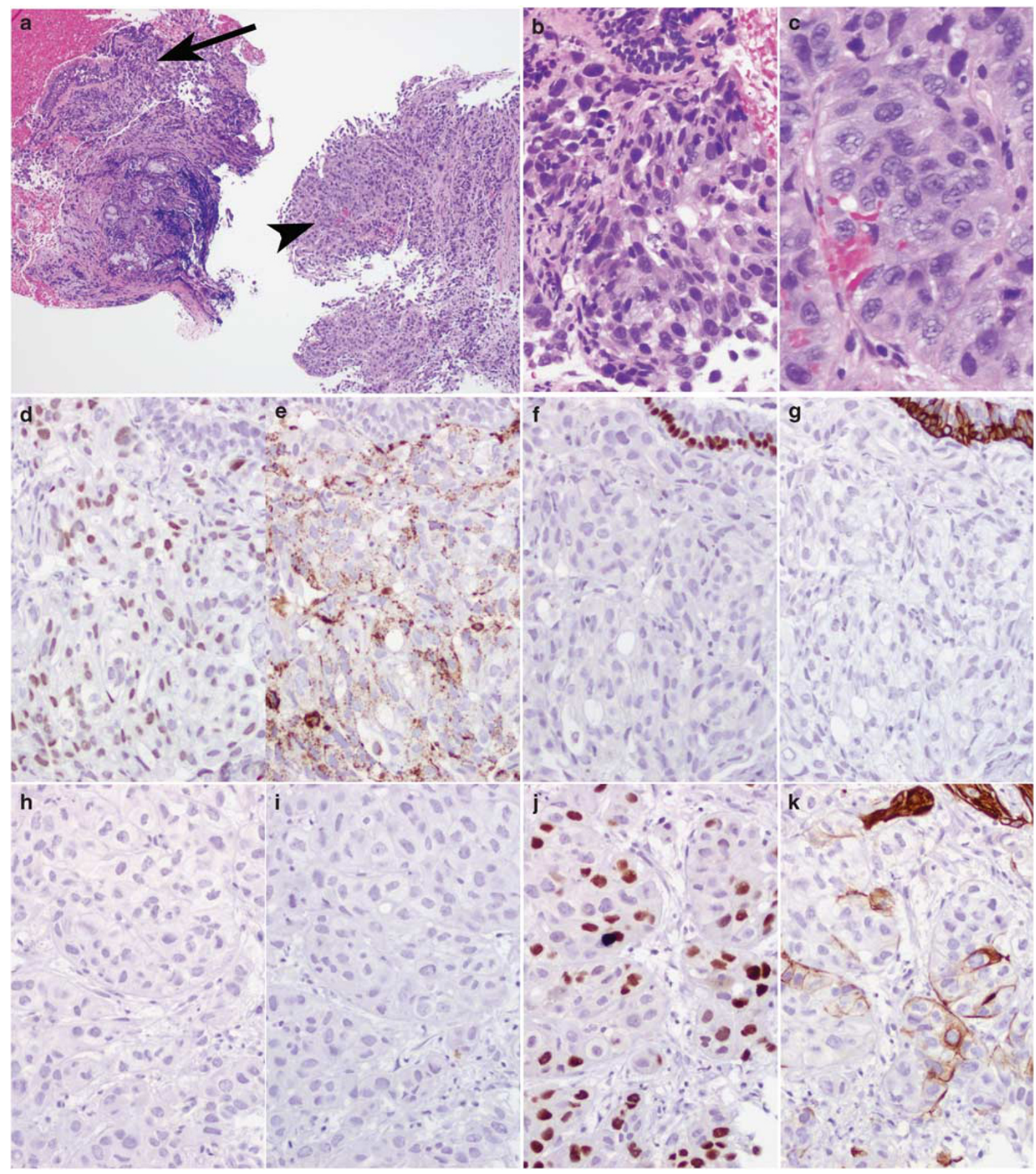

Figure 8 Immunohistochemical findings in poorly differentiated adenosquamous carcinoma in a small biopsy. (a) Endobronchial biopsy showing a tumor composed of two components, one just beneath the bronchial epithelium (long arrow) and the other in a separate fragment (arrowhead). (b) High magnification of the first component shows a poorly differentiated non-small cell carcinoma that cannot be classified on H\&E. (c) The second component is also poorly differentiated, although with cells that are slightly larger than the first component. (d-g) Immunohistochemical profile of the tumor in panel b. This component is positive for TTF-1 (panel d) and napsin A (panel e) but negative for p63 (panel f) and CK5/6 (panel g). (h-k) Immunohistochemical profile of the component in panel c, which is negative for TTF-1 (panel h) and napsin A (panel $\mathbf{i}$ ) but positive for p63 (panel $\mathbf{j}$ ) and CK5/6 (panel $\mathbf{k}$ ), indicating a true squamous component. The combination of these two components is diagnostic of a poorly differentiated adenosquamous carcinoma.

or napsin A-positive squamous cell carcinomas in the literature. It is also helpful to remember that the cytoplasm of alveolar macrophages stains positive for napsin A. Such cells, when present within tumor tissue, represent another potential source of misinterpretation. 

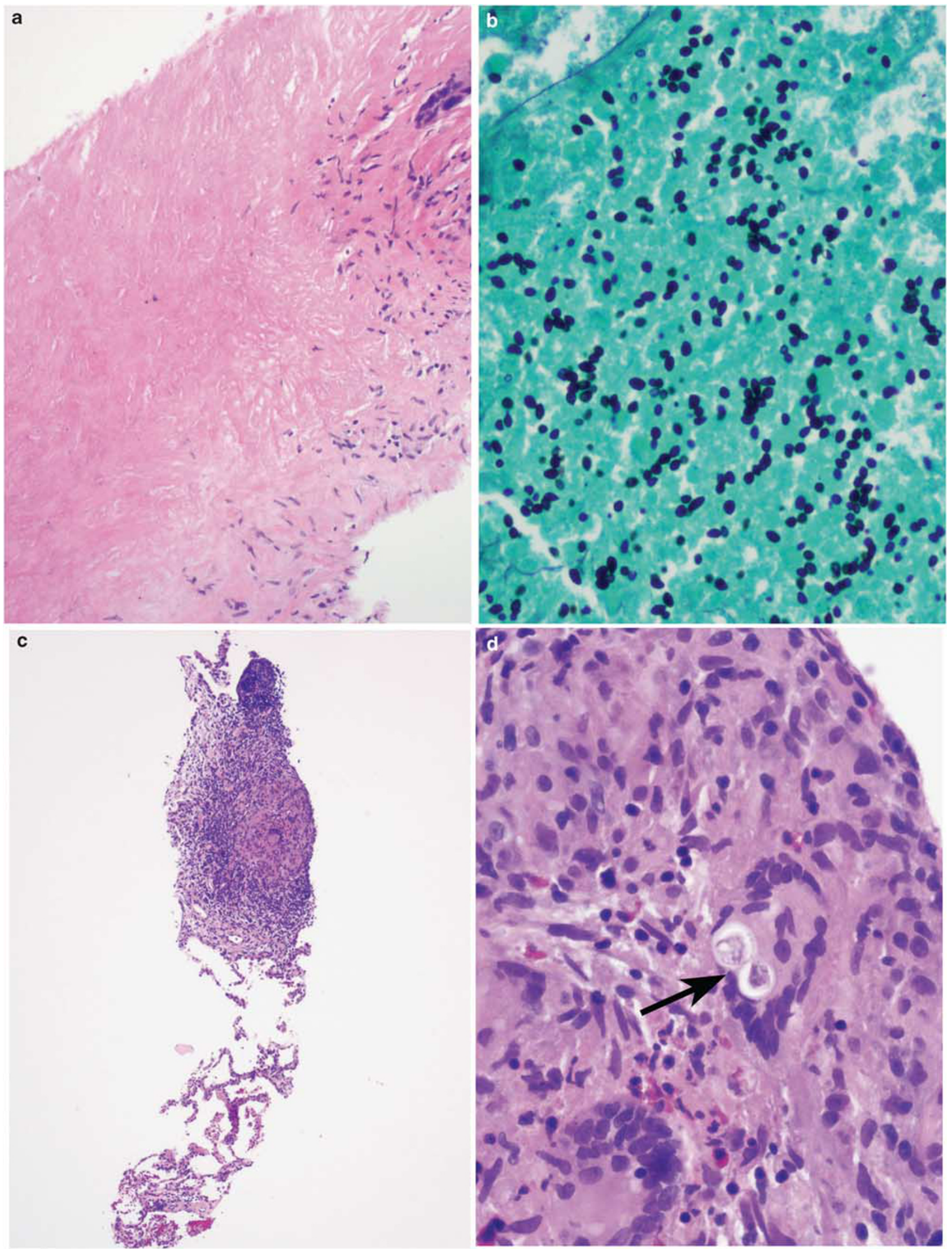


\section{Should tumors that stain for both TTF-1 and $\mathrm{p} 63$ be diagnosed as adenosquamous carcinoma?}

As shown in Table 1 and Figures 7 and 8, this combination can be encountered in two situations:

(1) The more common circumstance is for TTF-1 to be diffusely positive, whereas p63 is only focally positive. Importantly, p63 is focally expressed in the same population of cells that expresses TTF-1. In some cases, TTF-1 does not stain all tumor cells, but TTF-1-positive cells still exceed p63-positive cells. This pattern of staining is fairly common in poorly differentiated adenocarcinomas and should not be misinterpreted as evidence of adenosquamous carcinoma. ${ }^{5,7}$ In cases in which TTF-1 staining is not significantly different from p63 staining, positivity for napsin A can be very helpful to support a diagnosis of poorly differentiated adenocarcinoma (Figure 7). Diffuse positivity for both TTF-1 and p63 in the same population of cells is distinctly uncommon, but should also be interpreted as poorly differentiated adenocarcinoma, especially if napsin A is also positive. Diffuse coexpression of TTF-1 and p63 has been recently reported to be a common feature of adenocarcinomas with signet ring cells, many of which harbor EML4-ALK translocations. ${ }^{13}$

(2) Less common are tumors with two separate components characterized by totally opposite, mirror-image staining patterns. In one component, TTF-1 and napsin A are positive, whereas p63 and CK5/6 are negative. In the other, p63 and CK5/6 are positive, whereas TTF-1 and napsin A are negative. Such tumors are true adenosquamous carcinomas (Figure 8). Unlike the pattern described in poorly differentiated adenocarcinomas, TTF-1 and p63 do not stain the same population of cells. Either component may be differentiated enough to be recognized on $\mathrm{H} \& \mathrm{E}$, and thus may not require immunohistochemical stains for recognition. However, if one or both components are poorly differentiated (as in Figure 8), the use of immunohistochemistry is justified to clarify the diagnosis.

\section{Can all poorly differentiated non-small cell lung carcinomas be classified by immuno- histochemistry?}

No. Some poorly differentiated non-small cell lung carcinomas cannot be classified even after immuno- histochemical staining. ${ }^{5,6}$ Such cases account for approximately $23-27 \%$ of poorly differentiated nonsmall cell lung carcinomas, which equates to $\sim 7 \%$ of all non-small cell carcinomas. ${ }^{5,6}$ Typically, such cases are negative for all four markers in the panel. At resection, most are either poorly differentiated adenocarcinomas that do not express TTF-1 or napsin A, or true large cell carcinomas. On small biopsies, such tumors should be diagnosed as 'poorly differentiated non-small cell lung carcinoma', or 'non-small cell lung carcinoma, not otherwise specified' (NSCLC, NOS). ${ }^{11}$ The term 'large cell carcinoma' should not be used on a small biopsy, as by definition, this diagnosis requires examination of the entire resected tumor. From a clinical standpoint, tumors that are unclassifiable by immunohistochemistry remain candidates for pemetrexed therapy.

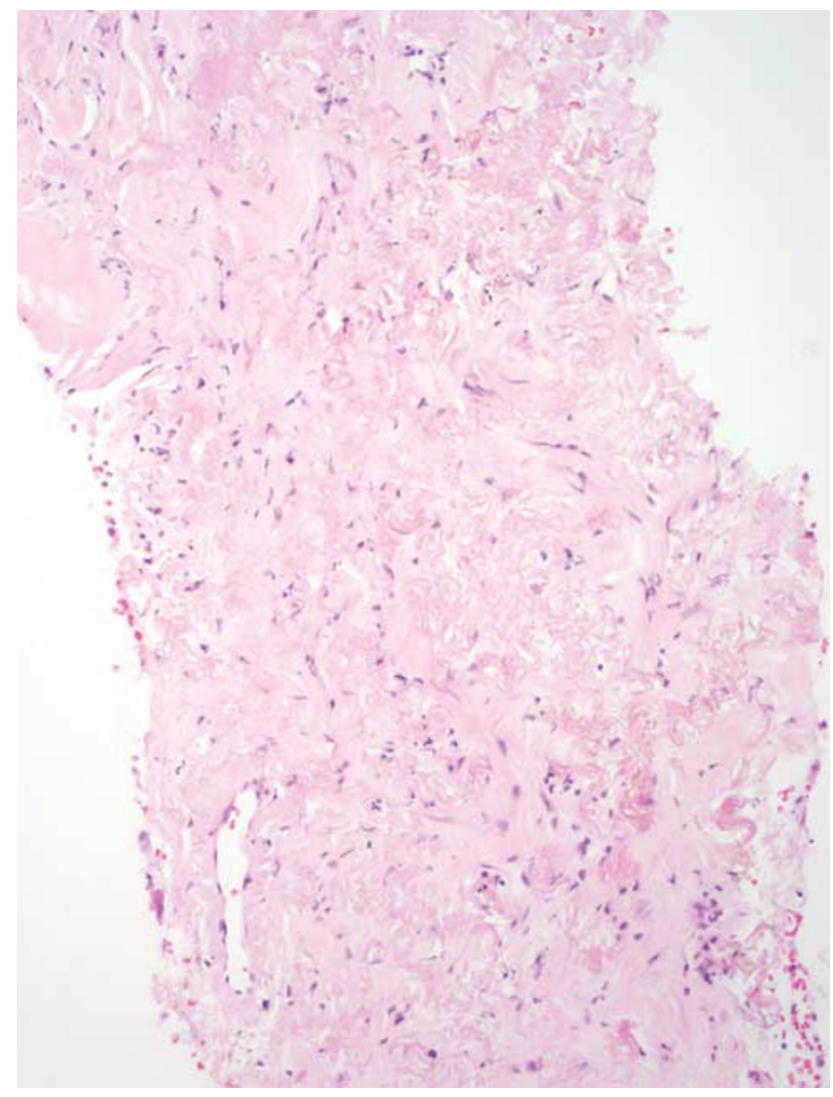

Figure 10 Scar. Core needle biopsy of a right apical lung lesion in a 50-year-old man with a history of small cell carcinoma treated with radiation and chemotherapy. The biopsy shows a scar, characterized by replacement of lung parenchyma by fibrosis. In contrast to the fibrosis illustrated in Figure 14, lung architecture is obliterated. The lesion remained stable over 4 years of radiological follow-up.

Figure 9 Granulomatous inflammation on core needle biopsies of lung nodules. (a) Core needle biopsy of a 1-cm nodule from a 71-yearold woman with a history of breast carcinoma shows a necrotizing granuloma. Necrosis is at the top left and granulomatous rim at the bottom right. (b) Same case as panel a. Grocott methanamine silver demonstrates numerous Histoplasma yeasts within the necrotic area. (c) Core needle biopsy of a lung lesion in a 46-year-old man shows granulomatous inflammation containing rare Blastomyces organisms within multinucleated giant cells (d). The broad-based budding (arrow) must be noted. 

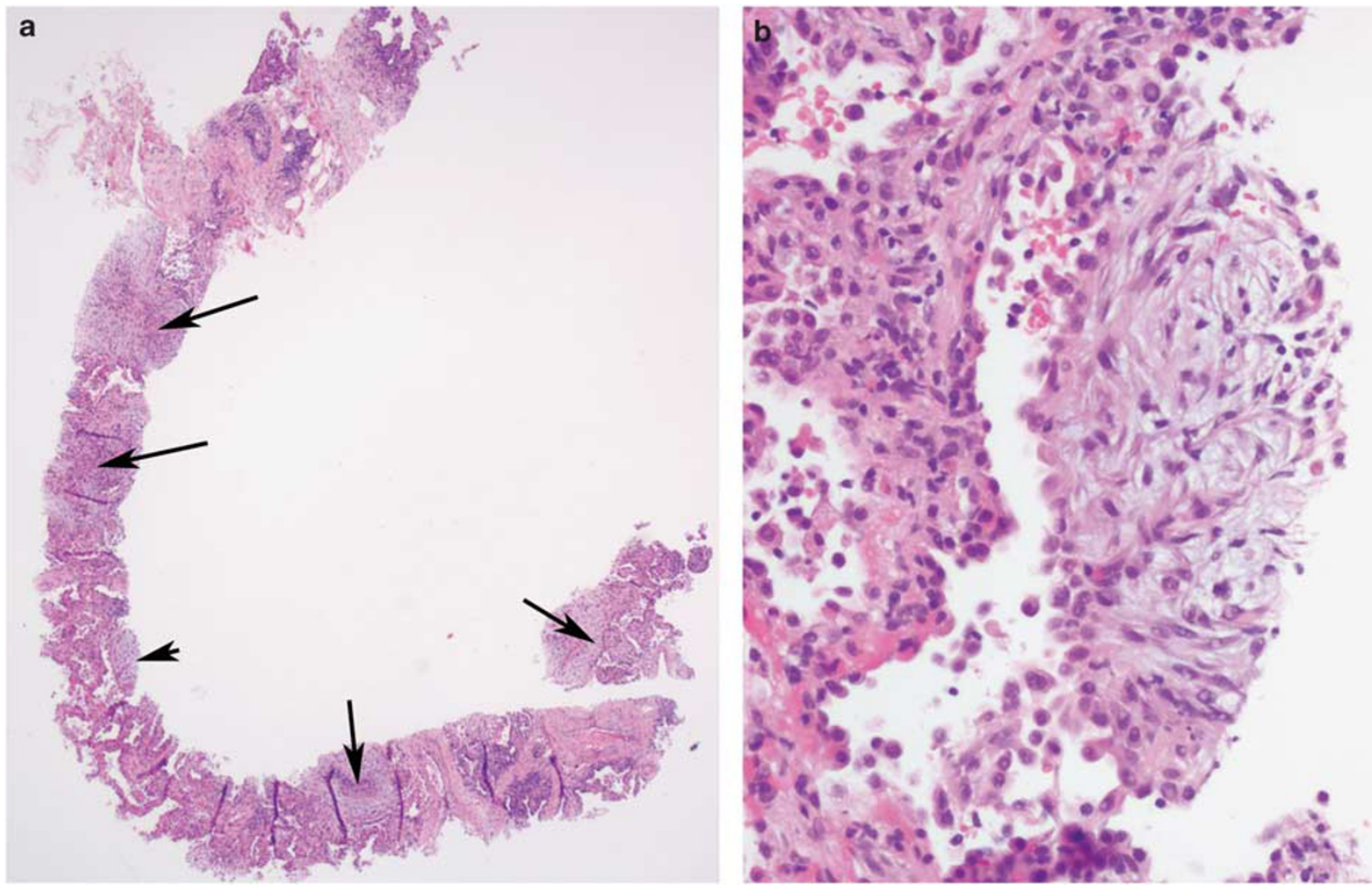

Figure 11 Organizing pneumonia. (a) Core needle biopsy of a 2.5-cm nodule in a 51-year-old male smoker found to have multiple nodules in the right lower lobe. The solid areas at low magnification are airspaces that are filled by fibroblast plugs (arrows and arrowhead). (b) High magnification of the fibroblast plug indicated by the arrowhead in panel a, showing fibroblasts within a pale-staining stroma.


Figure 12 Chondroid hamartoma. Core needle biopsy of a 1.3-cm lung nodule in a 53-year-old woman. (a) Characteristic admixture of adipose tissue (top arrow), cytologically bland spindle cells in a myxoid stroma (arrowhead) and entrapped lung epithelium (bottom arrow). (b) A fragment of cartilage was present elsewhere in the biopsy, with a transition to a spindle cell area (right). 


\section{Does immunohistochemistry exclude other primary or metastatic tumors from the differential diagnosis?}

No. The panel described above was designed for a single specific purpose, ie, to subclassify non-small cell lung carcinomas that cannot be subclassified using standard criteria on H\&E-stained slides. The panel is not a panacea for every situation and must be used only after a thorough evaluation of morphological features on H\&E-stained slides. Its use assumes that small cell carcinoma, large cell neuroendocrine carcinoma and other primary and metastatic malignant tumors have been excluded based on H\&E morphology, clinical history and radiological findings. The panel is not intended to differentiate primary lung tumors from metastases, as neither TTF-1 nor napsin A is perfectly sensitive or specific for pulmonary origin. Similarly, p63 and CK5/6 stain squamous cell carcinomas from virtually any primary site. TTF-1-positive tumors in the lung that are negative for napsin A, p63 and CK5/6 are not necessarily adenocarcinomas, as small cell carcinomas and large cell neuroendocrine carcinomas may have an identical immunohistochemical profile. Therefore, as in any other situation, the use of immunohistochemistry must be guided by H\&E morphology.

A question that is frequently asked in this context is whether immunohistochemical stains should be used for determining whether a carcinoma in the lung is a primary carcinoma or a metastasis. It is important to keep in mind that most solitary lung nodules that prove to be carcinomas on biopsy are primary lung carcinomas. Conversely, most patients with lung metastases have a known history of a primary elsewhere. Therefore, the use of immunohistochemistry should be restricted to situations in which there is a good reason to suspect a metastatic tumor, such as a known history of carcinoma elsewhere, multiple lung nodules on radiographs or unusual H\&E morphological features suggestive of an alternative primary. Outside these situations, the use of immunohistochemistry to 'confirm' or 'exclude' a lung primary is of dubious value and may result in unnecessary work-up and diagnostic delay. Markers such as CK7 and TTF-1 are not specific for lung origin, and merely provide false reassurance. Furthermore, negative staining for these markers does not exclude a lung primary.

\section{Can specific benign lesions be accurately diagnosed on core biopsies of lung nodules?}

Yes. One of the major advantages of core biopsies over fine-needle aspirates for the diagnosis of lung nodules is that they increase the likelihood of a specific benign diagnosis when compared with fineneedle aspiration, ${ }^{14}$ obviating the need for additional sampling in most cases. ${ }^{15}$

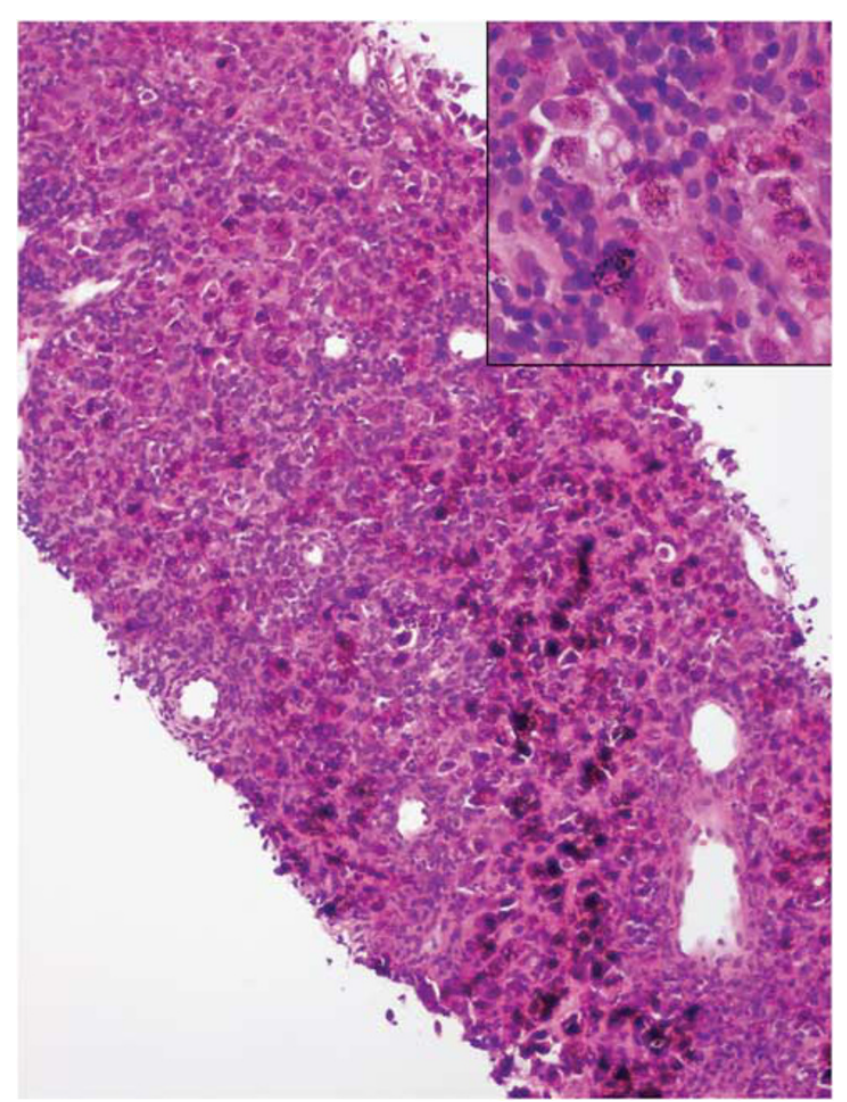

Figure 13 Intrapulmonary lymph node. Core needle biopsy of a 6 -mm nodule in the right lower lobe of the lung in a 68-year-old smoker. The biopsy shows a benign lymph node containing lymphocytes and numerous dust-laden macrophages (inset).

The most common specific benign diagnosis in core biopsies of lung nodules is granulomatous


granulomas, a third of which can be shown to contain mycobacterial or fungal organisms (Figure 9). Parenchymal scars are the next most common diagnosis. They are characterized by replacement of lung architecture by fibrosis (Figure 10). Scars may be caused by previous radiation or may represent so-called apical caps. Organizing pneumonia (Figure 11) is another common benign diagnosis on core biopsies. ${ }^{16}$ It is characterized by filling of airspaces by fibroblast plugs. In order for this diagnosis to be considered specific, fibroblast plugs should be present in all or most of the involved core. Benign lung neoplasms are uncommon but can be reliably diagnosed on core biopsies of lung nodules. The most common are chondroid hamartoma (Figure 12), solitary fibrous tumor and schwannoma. Other specific benign diagnoses that can be rendered on core biopsies include abscess, nodular amyloidosis, intrapulmonary lymph node (Figure 13), mycetoma, invasive fungal pneumonia, aspiration pneumonia and cryptococcal pneumonia. 


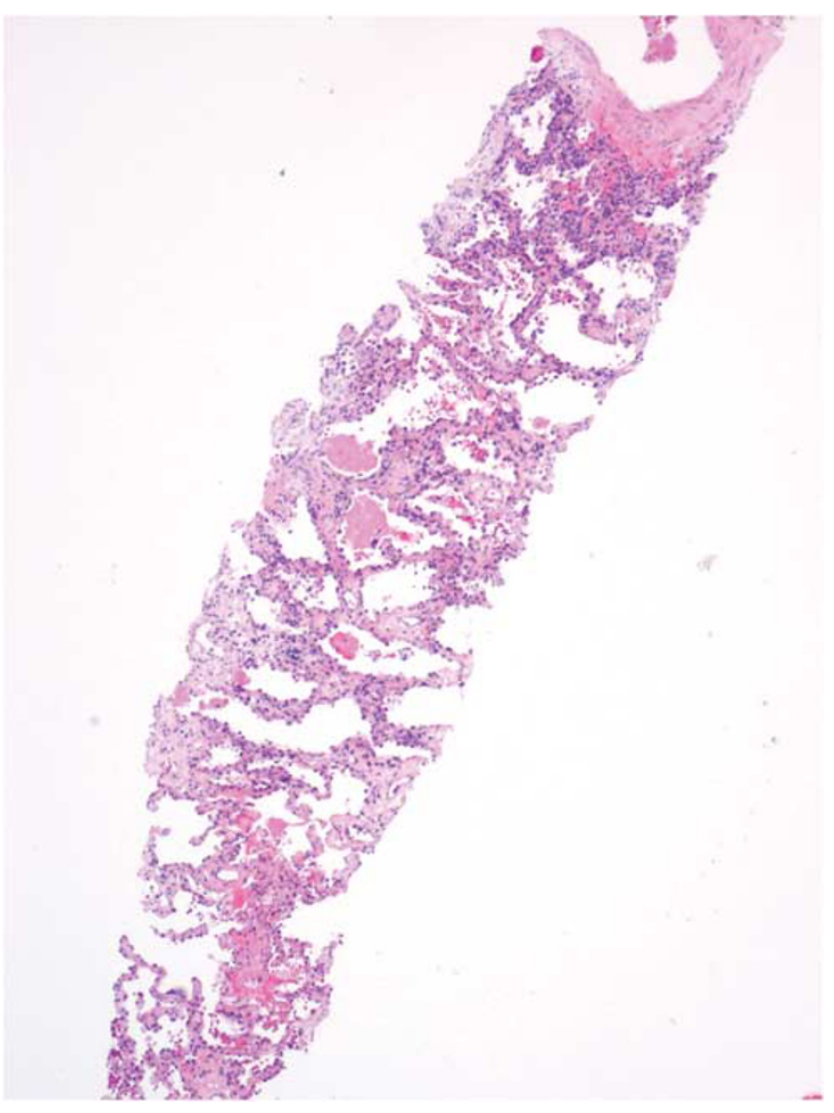

Figure 14 Non-specific finding on core needle biopsy of lung. Core biopsy of a 1-cm lung PET-positive nodular airspace opacity in a 49-year-old woman. The interstitium is mildly expanded by fibrosis and chronic inflammation, but lung architecture remains intact. In contrast to fibrous scars (see Figure 10), this type of fibrosis is a non-specific finding. This lesion remained stable at 18 months of radiological follow-up.

Langerhans cell histiocytosis is a form of smokingrelated interstitial lung disease that is characterized radiologically by multiple tiny bilateral nodules. This condition is usually diagnosed on surgical lung biopsies. However, transbronchial biopsies can also be diagnostic in some cases. As the nodules of Langerhans cell histiocytosis are tiny, it is generally assumed that they cannot be sampled by a core needle biopsy. However, a recent report described three cases, all with subcentimeter nodules, which were diagnosed on CT-guided percutaneous core biopsies, sparing the patients an invasive surgical procedure. ${ }^{17}$

\section{In core biopsies of lung nodules that lack malignant cells, what is the significance of non-specific findings?}

In some cases, core biopsies lack malignant cells and show only non-specific histological findings such as interstitial fibrosis and chronic inflammation (Figure 14), necrosis, acute and chronic inflammation, intra-alveolar fibrin or organizing hemorrhage.
Such lesions differ from specific entities such as parenchymal scars in that they do not cause significant architectural distortion and therefore do not adequately account for the presence of a nodule. Although it is possible that such findings may be representative of the target lesion, in contrast to the specific diagnoses mentioned in the previous section, such non-specific findings should not be considered diagnostic, and this should be communicated to the clinician in a comment. Small, fragmented biopsies are more likely to be nondiagnostic. ${ }^{15}$ The further course of action in such cases depends on clinical and radiographic findings. Nodules that progressively increase in size or are suspicious for malignancy because of other reasons should be re-biopsied if the initial biopsy shows non-specific findings only. On the other hand, if the clinical index of suspicion is low, these patients can be followed radiologically.

\section{Summary and take-home points}

Hopefully, this review will serve as a practical guide to pathologists who are increasingly called to 'do more with less' in the diagnosis of lung nodules. Even in small biopsies, most non-small cell lung carcinomas can be subclassified on H\&E-stained slides, and immunohistochemistry is required only for cases that lack glands, mucin, intercellular bridges or keratinization. The best panel for subclassifying such tumors is TTF-1, napsin A, p63 and CK5/6. Core needle biopsies are useful not only for detecting malignant cells, but also for diagnosing specific benign lesions such as granulomas, scars and organizing pneumonia, obviating the need for further sampling in most cases. Repeat sampling in cases with non-specific findings should be based on clinical features.

\section{Disclosure/conflict of interest}

This author declares no conflict of interest.

\section{References}

1 Scagliotti GV, Parikh P, von Pawel J, et al. Phase III study comparing cisplatin plus gemcitabine with cisplatin plus pemetrexed in chemotherapy-naive patients with advanced-stage non-small-cell lung cancer. J Clin Oncol 2008;26:3543-3551.

2 Shigematsu H, Lin L, Takahashi T, et al. Clinical and biological features associated with epidermal growth factor receptor gene mutations in lung cancers. J Natl Cancer Inst 2005;97:339-346.

3 Johnson DF, Fehrenbacher L, Novotny WF, et al. Randomized phase II trial comparing bevacizumab plus carboplatin and paclitaxel with carboplatin and paclitaxel alone in previously untreated locally advanced or metastatic non-small-cell lung cancer. J Clin Oncol 2004;22:2184-2191. 
4 Kwak EL, Bang YJ, Camidge DR, et al. Anaplastic lymphoma kinase inhibition in non-small-cell lung cancer. N Engl J Med 2010;363:1693-1703.

5 Mukhopadhyay S, Katzenstein AL. Subclassification of non-small cell lung carcinomas lacking morphologic differentiation on biopsy specimens: utility of an immunohistochemical panel containing TTF-1, napsin A, p63 and CK5/6. Am J Surg Pathol 2011;35:15-25.

6 Loo PS, Thomas SC, Nicolson MC, et al. Subtyping of undifferentiated non-small cell carcinomas in bronchial biopsy specimens. J Thorac Oncol 2010;5: 442-447.

7 Rekhtman N, Ang DC, Sima CS, et al. Immunohistochemical algorithm for differentiation of lung adenocarcinoma and squamous cell carcinoma based on large series of whole-tissue sections with validation in small specimens. Mod Pathol 2011;24:1348-1359.

8 Righi L, Graziano P, Fornari A, et al. Immunohistochemical subtyping of nonsmall cell lung cancer not otherwise specified in fine-needle aspiration cytology. A retrospective study of 103 cases with surgical correlation. Cancer 2011;117:3416-3423.

9 Bishop JA, Sharma R, Illei PB. Napsin A and TTF-1 expression in carcinomas of the lung, breast, pancreas, colon, kidney, thyroid, and malignant mesothelioma. Hum Pathol 2010;41:20-25.

10 Yang M, Nonaka D. A study of immunohistochemical differential expression in pulmonary and mammary carcinomas. Mod Pathol 2010;23:654-661.

11 Travis W, Brambilla E, Noguchi M, et al. International Association for the Study of Lung Cancer/American
Thoracic Society/European Respiratory Society international multidisciplinary classification of lung adenocarcinoma. J Thorac Oncol 2011;6:244-285.

12 Nicholson AG, Gonzalez D, Shah P, et al. Refining the diagnosis and EGFR status of non-small cell lung carcinoma in biopsy and cytologic material, using a panel of mucin staining, TTF-1, cytokeratin 5/6, and p63, and EGFR mutation analysis. J Thorac Oncol 2010;5:436-441.

13 Yoshida A, Tsuta K, Watanabe S, et al. Frequent ALK rearrangement and TTF-1/p63 co-expression in lung adenocarcinoma with signet-ring cell component. Lung Cancer 2011;72:309-315.

14 Gong Y, Sneige N, Guo M, et al. Transthoracic fineneedle aspiration vs. concurrent core needle biopsy in diagnosis of intrathoracic lesions: a retrospective comparison of diagnostic accuracy. Am J Clin Pathol 2006;125:438-444.

15 Doxtader EE, Mukhopadhyay S, Katzenstein AL. Core needle biopsy in benign lung lesions: pathologic findings in 159 cases. Hum Pathol 2010;41: 1530-1535.

16 Aviram G, Greif J, Man A, et al. Diagnosis of intrathoracic lesions: are sequential fine-needle aspiration (FNA) and core needle biopsy (CNB) combined better than either investigation alone? Clin Radiol 2007;62: 221-226.

17 Mukhopadhyay S, Eckardt SM, Scalzetti EM. Diagnosis of pulmonary Langerhans cell histiocytosis by CTguided core biopsy of lung: a report of three cases. Thorax 2010;65:833-835. 